\title{
EFFECT OF NUTRIENTS ON ENVIRONMENTAL SEX DETERMINATION AND SIZE OF GAMETOPHYTES IN CULCITA MACROCARPA
}

\author{
Lipika Ghosh $^{{ }^{* 1}}$, Ares Jiménez ${ }^{1}$, Luis G. Quintanilla ${ }^{1}$ \\ ${ }^{1}$ Departamento de Biologíay Geología, ESCET, Universidad Rey Juan Carlos, E-28933 Móstoles, Madrid, Spain. \\ *Department of Botany, University of Rajshahi, Rajshahi-6205, Bangladesh. E-mail: lipika_ru@yahoo.com
}

\begin{abstract}
In environmental sex determination (ESD) gender is decided after conception, depending on the environment, rather than being genetically fixed. ESD has rarely been studied in homosporous ferns. In the present study, Culcita macrocarpa gametophytes were cultured under varying nutrient conditions. Initially, most of the gametophytes of Culcita macrocarpa were male and subsequently hermaphrodite under different nutrition. The result indicates that its sex determination is protandry. All nutrient conditions were favourable for developing male prothalli but only good environment (high nutrient) was favourable for female gametophyte growth. In all respects, female gametophytes were much larger, than the other types of gametophytes. Hermaphroditic gametophytes were larger than male gametophytes, which were larger than asexual gametophytes.
\end{abstract}

Key Words: Environmental sex determination, homosporous fern, Culcita macrocarpa, protandry, hermaphroditic.

সারাংশ ঃ পরিবেশীয় লিঙ্গ নির্ধারণের (ESD) ক্ষেত্রে গর্ভধারণের পর লিঙ্গ নির্ধারণ জীনগত নির্ধারণের চেয়ে পরিবেশীয় নির্ধারণের উপর বেশী নির্ভরশীল। সমরেণুপ্রসূ ফার্ণে পরিবেশীয় লিঙ্গ নির্ধারণ সম্পর্কে কিছু গবেষণা হয়েছে। বর্তমান গবেষণায় বিভিন্ন পুষ্টিক্ষেত্রে Culcita macrocarpa এর চাষ করা হয়েছে। প্রাথমিক পর্যায়ে বিভিন্ন পুষ্টিগত অবস্থায় C. macrocarpa এর অধিকাংশ লিঙ্গের উদ্ভিদ পুরুষ এবং পরবর্তীতে উভলিঙ্গ। ফলাফল নির্দেশ করে যে,এদের লিঙ্গ নির্ধারণ প্রোটান্দ্রি (Protandry)। সকল পুষ্টি মাধ্যম পুং প্রোথেলাসের জন্য উপযুক্ত কিন্তু শুধুমাত্র ভাল পরিবেশই স্ত্রী লিঙ্গধরের জন্য উপযুক্ত। সকল ক্ষেত্রে, স্ত্রী লিঙ্গধর অন্য সকল লিঙ্গধর উদ্ভিদের তুলনায় আকারে অনেক বড়। উভলিঙ্গ লিঙ্গধর পুং-লিঙ্গধর থেকে বড় যা আবার অযৌন লিঙ্গধর থেকে বড়।

\section{Introduction}

Mechanisms of sex determination in plants have attracted much attention within plant biology (Tanurdzic and Banks 2004). Environmental sex determination is particularly interesting for physiological, ecological and evolutionary reasons. Environmental sex determination is a form of phenotypic plasticity, where individuals produce either female, male or both sex organs depending largely upon environmental circumstances (Leimar et al. 2004). Evolutionary theory predicts that environmental sex determination is selected for when a factor that varies across the environment differentially and influences female and male performance (Charnov and Bull 1977). Environmental sex determination is widespread in many animal and plant taxa (Freeman et al. 1980 and Korpelainen, 1998), but only in homosporous pteridophytes it becomes dominant. Homosporous pteridophytes produce a single kind of spore that develops into potentially bisexual gametophytes. However, in many species actual gender is conditional on environmental conditions. Gametophytes of many species tend to become male under poor growing conditions, such as poor light level and quality (Guillon and Fievet 2003), low nutrient availability (Korpelainen, 1994) or high density (Huang et al. 2004). Conversely, gametophytes normally become female under rich growing conditions. A theoretical basis for the relationship between stressing conditions, reduced growth rate and maleness comes from an evolutionary model proposed by Haig and Westoby (1988). Based on a higher cost of female reproduction and other premises, they predicted female sex expression under rich growing conditions and a tendency to reproduce as a male under poor conditions.
Culcita macrocarpa C. Presl is the only European member of the family Dicksoniaceae with very large fronds, often over $2 \mathrm{~m}$ long that are persistent in winter and arranged in crowns (Figure 1). The spores of this species are highly sensitive to desiccation (Quintanilla et al. 2002). In northwest Spanish populations of $C$. macrocarpa sporangium dehiscence occurs around the spring equinox, when temperatures are suitable for germination (Quintanilla et al. 2000).



Figure 1 Sporophyte of Culcita macrocarpa.

The fern Culcita macrocarpa produces only one type of spore (i.e., homosporous), yet each Culcita spore has the potential to develop as a free-living haploid green gametophyte with numerous rhizoids on the inferior central surface cushion (Figure 2) which are sexually protandrous (Quintanilla et al. 2005) as like as Ceratopteris (Banks 1997 1999) where the sex of the gametophyte is determined during the development of only sperm or egg or produces both egg and sperm. 


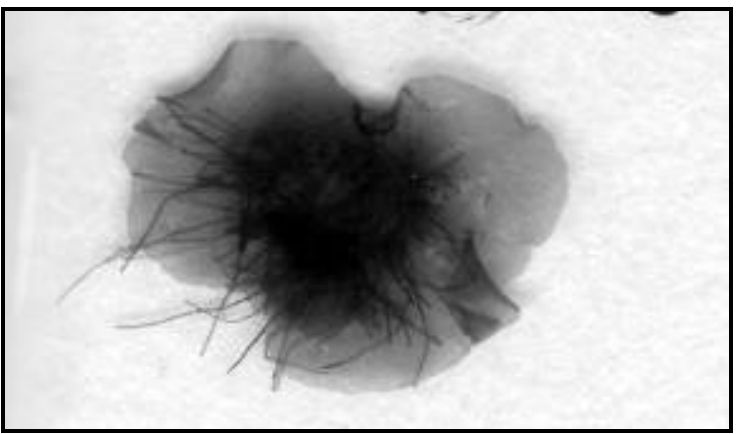

Figure 2 Gametophyte of Culcita macrocarpa.

Sex differentiation is manifested in the increasing complexity of interaction during nutrient supply, the retention of the gametophyte. Carbohydrates are known to perform important regulatory functions in the plant life cycle, including photosynthesis (Lloyd and Zakhleniuk, 2004; Halford and Paul 2003) and carbohydrate partitioning (Rook and Bevan 2003) mechanisms by using different sugars to influence the gene expression and ultimately plant development (Gibson, 2005).

Stress induced sexual expression in fern gametophytes was fruitfully studied within the ESD theoretical framework by Lucía DeSoto et al. (2008) and found the effects of nutrient availability and individual density in Woodwardia radicans. Nutrients as well as other factors are implicated in the growth and development of gametophytes and sporophytes (Fernández et al. 1999). Although references to such environmental influences on gametophyte gender are scanty, it is only recently that an ESD-based interpretation has gained momentum.

In this study, the effect of nutrition on sex determination was assessed to establish whether this species fits the model of Haig and Westoby (1988). Growth of C. macrocarpa was also examined to provide information on the size of gametophytes needed to favour successful reproduction.

\section{Material and Methods}

C. macrocarpa gametophytes were cultured under varying nutrient conditions. Spores were sown under three different nutrient levels (Dyer agar diluted 0,100 and 1000 times). The fixed level of light (55-65 PARS) was used for this experiment.

Seven weeks after sowing, 200 gametophytes per nutrient level were transplanted into individual cell under the same nutrient conditions for germination. Cultures were incubated in a phytothron with a $14 \mathrm{~h}$ photoperiod and alternating $20{ }^{\circ} \mathrm{C}$-light $/ 15^{\circ} \mathrm{C}$-darkness temperatures.

To elucidate patterns of gender expression in gametophytes, 100 gametophytes per nutrient level were randomly sampled. Gametophytes were stained with acetocarminechloral hydrate after 19 and 27 weeks of sowing and their sex and number of gametangia were determined under a light microscope. To determine gametophyte size, all individuals were scanned and their area was measured with the program Image (Abramoff et al. 2004).

\section{Results and Discussion}

In this study, the same culture conditions were applied and the developmental differences were observed between two times (19 and 27 weeks).

Antheridia formed on the dorsal and ventral surfaces of the wing, while archegonia formed on the ventral surface of the anterior cushion. In all cultures, the mature antheridium was globose and consisted of a basal cell, a cylindrical ring and a cap cell (Figure 3). Archegonia had long neck cells (Figure 4).

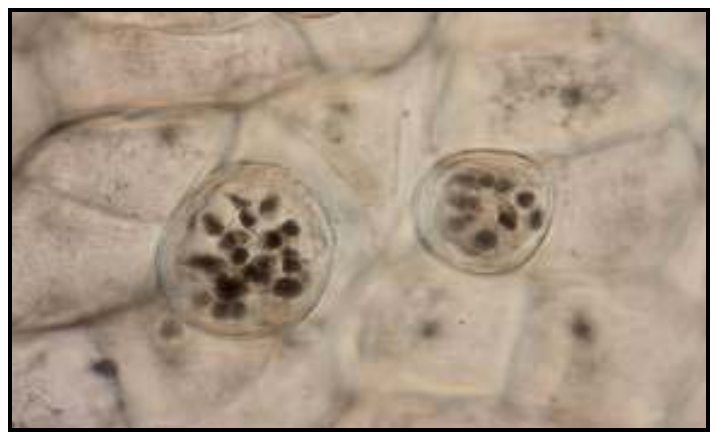

Figure 3 Antheridia of Culcita macrocarpa.

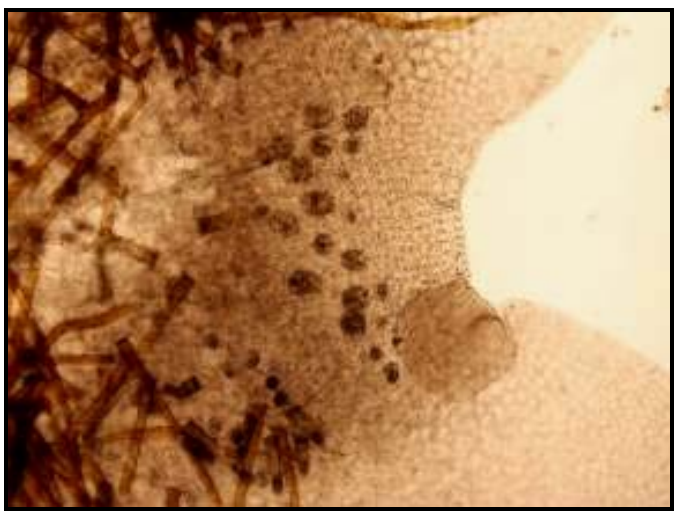

Figure 4 Archegonia of Culcita macrocarpa.

Gender was found to be significantly affected by nutrient level. After sowing, the gametophytes of Culcita macrocarpa (Figure 2) exhibited successful vegetative and reproductive growth conditions overall.

Antheridia appeared first in all nutrients after 19 weeks of sowing. Most of the gametophytes became male and were found to be positioned about more than half except $1 / 100$ nutrient levels but after 27 weeks, male percentages decreased at 1/100 and undiluted nutrient levels. Gametophytes showed faster growth, especially with mid and high nutrient levels. Female and bisexual 
became predominant at that time and reached female $30 \%$ and $10 \%$, and bisexual $65 \%$ and $71 \%$ at $1 / 100$ and undiluted nutrient levels, respectively.

In the first time, laboratory populations were mostly antheridiate and bisexuals but in the second time antheridiate with archegoniate and bisexuals.

Most of the gametophytes of the fern Culcita are either male or hermaphrodite. In some homosporous fern that has no antheridiogen activity, their sex is epigenetically determined by the environmental factors. The male and hermaphroditic gametophytes of Culcita are morphologically distinct by the type of gametangia produced and as well as by shape and size. Hermaphroditic gametophytes have a distinct lateral multicellular meristem from which most cells of the hermaphrodite prothallus are derived. This meristem forms a meristem notch that gives the hermaphrodite its heart-shaped appearance (Strain et al. 2001). The male gametophyte is much smaller and usually shorter-lived than the hermaphrodite (Bank 1997). Hermaphrodites produce both antheridia and archegonia; they can be self-fertilized to form homozygous sporophytes.

To understand fern reproduction, it is necessary to understand sexual expression and the factors that affect it.
Although homosporous ferns have the potential to be hermaphroditic, many factors may affect their growth and sexual expression (Verma and Selvan, 2001). In the present study gametophytes of $C$. macrocarpa were initially male and subsequently hermaphrodite under the different nutritions level. The data obtained, regarding their sex determination was protandry, which is in agreement with the previous research (Quintanilla et al. 2005).

Gametophytes of $C$. macrocarpa develop almost exclusively as males, in the absence of antheridiogen and then hermaphrodites. The male prothalli expression is stable and more or less indifferent in all times on sex determining signals once its sex is established. In this connection it can be said that all males are genotypically developed. Because the number of male prothalli did not change even after developing archegonia but asexual gametophytes decreased with time. So from this view, it can be assumed that female prothalli is developed by changing of asexual gametophytes not from male gametophytes. Both conditions are favourable for developing of male prothalli but only good environment is favourable for female gametophyte growth, which supports the ESD theory. Because, here good environment is responsible for archegonia development by which breeding system can be changed.



Figure 5 Relative frequencies of gametophyte genders at the three nutrient levels.

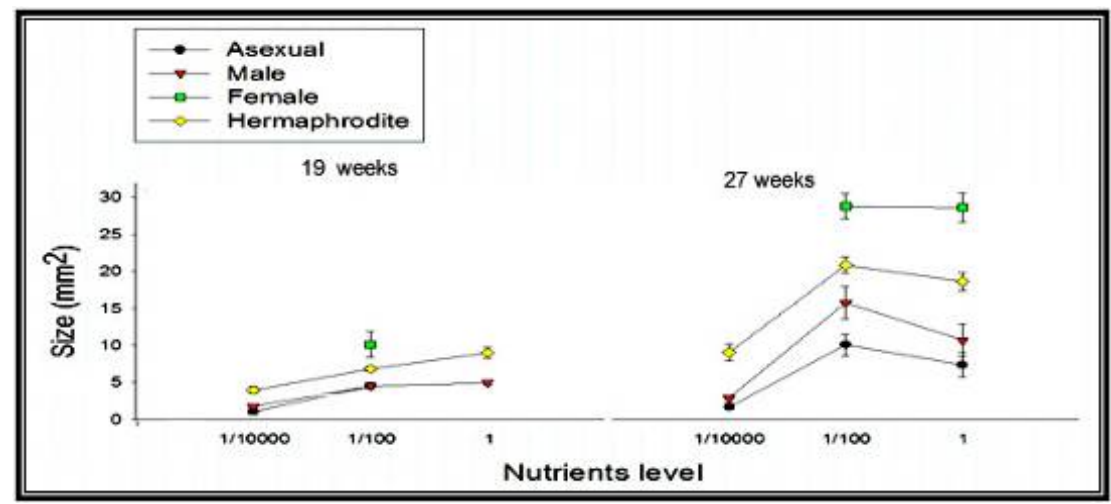

Figure 6 Gametophyte size at the three nutrient levels. 
In all respects, female gametophytes were much larger than other types of gametophytes. Hermaphroditic gametophytes were larger than male gametophytes, which were larger even than asexual gametophytes. The size of females and bisexuals increased with increasing the nutrient level.

The size of C. macrocarpa in all samples was more or less similar in each kind of nutrition level. Consistently, all gametophytes grew well; the male gametophytes were always smaller than the female and bisexual ones. Similar result has been reported for other genera (Haufler and Ranker 1985). In female gametophytes, the number of archegonia significantly increased with gametophyte size across the two harvest times (Figure 5 \& 6). In bisexual gametophytes, archegonia and total gametangia significantly increased with gametophyte size. Males did not show clear size patterns number across nutrient levels.

Females were always larger than the males with significant assortment. Size and number were more important for females than for males in all phases of the breeding season. It was also compatible with the observations of earlier investigators (Naf 1956) that 'male' prothalli were smaller than 'female' prothalli and that conditions made interference with growth, e.g., low light intensity, crowding and poor mineral supply favoured the production of antheridia over that of archegonia.

Environmental factors affecting gender in ferns, such as nutrients, have received very little attention in most reviews regarding sex expression (Korpelainen 1998; Raghavan 1989,). In many fern species, immature gametophytes become female or male under rich or poor growth conditions, respectively. Resource of population is one of the factors affecting sexual expression and growth in gametophytic plants. Population resources are limited by competition, and gametophytes are often asexual or male, and narrow. On the other hand female and hermaphroditic gametophytes often occur in sparse populations (Cousens 1979). Plant growth and development ultimately depend upon environmental variables, such as temperature, light intensity and the availability of water and essential minerals (Marschner 1995). The longer time was required for successful female reproduction, compared to that of the fast male reproduction (Delph 1999).

\section{Conclusion}

Sexual expression in fern gametophytes can be fruitfully studied within the ESD theoretical framework. C. macrocarpa is first male and then hermaphrodite, even in conditions supposed to be optimal for growth. Natural populations of C. macrocarpa are presumably androdioecious (Quintanilla et al. 2005). Klekowski and Lloyd (1968) said that this sequence favours intragametophytic selfing. Size-related patterns of sexual expression were consistent with the size- advantage model, because female function benefited more from a larger size than did male function. The results of this study raise the possibility that certain morphological features, particularly the growth and sex expression of the gametophyte are controlled by the same regulatory mechanisms and genes that control environmental factors.

Acknowledgements: Authors are grateful to the Director of the Department of Biology and Geology of Rey Juan Carlos University for providing necessary facilities of the controlled temperature room and laboratory. This research was financially supported by Beca MAEC-AECI Grant, 2007-08, International Cooperation Spanish Agency.

\section{References}

Abramoff MD, Magelhaes PJ and Ram SJ. 2004. Image processing with Image. J. Biophotonics International 11: 36-42.

Banks JA. 1997. The transformer genes of the fern Ceratopteris simultaneously promote meristem and archegonia development and repress antheridia development in the developing gametophyte. Genetics 147: 1885-1897.

Banks JA. 1999. GAMETOPHYTE DEVELOPMENT IN FERNS. Plant Biology 50: 163-186

Charnov EL and Bull JJ. 1977. When is sex environmentally determined? Nature 266: 828-830.

Cousens MI. 1979. Gametophyte ontogeny, sex expression, and genetic load as measures of population divergence in Blechnum spicant. American Journal of Botany 66: 116-132.

Delph LF. 1999. Sexual dimorphism in life history. Gender and Sexual Dimorphism in Flowering Plants (eds M.A. Geber, T.E. Dawson \& L.F. Delph) Springer-Verlag pp. 149-173. Berlin.

Fernández H, Bertrand AM and Sanchez-Tames R. 1999. Biological and nutritional aspects involved in fern multiplication. Plant Cell Tissue and Organ Culture 56: 211-214.

Freeman DC, Harper KT and Charnov EL. 1980. Sex change in plants: Old and new observations and new hypotheses. Oecologia 47: 222-232.

Gibson SI. 2005. Control of plant development and gene expression by sugar signaling. Plant Biology 8(1): 93-102.

Guillon JM and Fievet D. 2003. Environmental sex determination in response to light and biased sex ratios in Equisetum gametophytes. Journal of Ecology 91: 49-57.

Haig D and Westoby M. 1988. Sex expression in homosporous ferns: an evolutionary perspective. Evolutionary Trends in Plants 2: 111-119.

Halford NG and Paul MJ. 2003. Carbon metabolite sensing and signalling. Plant Biotechnol. J. 1: 381-398.

Haufler CH and Ranker TA. 1985. Differential antheridiogen response and evolutionary mechanisms in Cystopteris. Am. J. Bot 72: 659-665.

Huang YM, Chou HM and Chiou WL. 2004. Density affects gametophyte growth and sexual expression of Osmunda cinnamomea (Osmundaceae: Pteridophyta). Annals of Botany 94: 229-232. 
Klekowski JR EJ and Lloyd RM. 1968. Reproductive biology of the Pteridophyta.I. General considerations and a study of Onoclea sensibilis L.Botanical Journal of the Linnean Society 60: 315-324.

Korpelainen H. 1994. Growth, sex determination and reproduction of Dryopteris filix-mas (L.)

Schott gametophytes under varying nutritional conditions. Bot. J. Linn. Soc. 114: 357-366.

Korpelainen, H. 1998. Labile sex expression in plants. Biological Reviews 73: 157-180.

Leimar O, Van Dooren TJM and Hammerstein P. 2004. Adaptation and constraint in the evolution of environmental sex determination. Journal of Theoretical Biology 227: 561-570.

Lloyd RM and Zakhleniuk OV. 2004. Responses of primary and secondary metabolism to sugar accumulation revealed by microarray expression analysis of the Arabidopsis mutant, pho3 J. Experimental Botany 55: 1221-1230.

Lucía DeSoto, Quintanilla LG and Méndez M. 2008. Environmental sex determination in ferns: effects of nutrient availability and individual density in Woodwardia radicans. Journal of Ecology 96(6): 1319-1327.

Marschner H. 1995. Mineral nutrition of higher plants. $2^{\text {nd }}$ Edition. Academic Press. San Diego. 889 pp.

Naf U. 1956. The demonstration of a factor concerned with the initiation of antheridia in polypodiaceous ferns. Growth 20: 91-105.
Quintanilla LG, Amigo J, Pangua E and Pajaro'n S. 2002. Effect of storage method on spore viability in five globally threatened fern species. Ann. Bot. 90: 461-467.

Quintanilla LG, Pajaro'n S, Pangua E and Amigo J. 2000. Effect of temperature on germination in northernmost populations of Culcita macrocarpa and Woodwardia radicans. Plant Biology 2: 612-617.

Quintanilla LG, Pangua E, Amigo J and Pajaro'n S. 2005. Comparative study of the sympatric ferns Culcita macrocarpa and Woodwardia radicans: sexual phenotype. Flora 200: 187-194.

Raghavan V. 1989. Developmental Biology of Fern Gametophytes. Cambridge University Press, Cambridge.

Rook F and Bevan M. 2003. Genetic approaches to understanding sugar-response pathways. J Experimental Botany 54: 495-501.

Strain E, Hass B and Banks J. 2001. Characterization of mutations that feminize gametophytes of the fern Ceratopteris. Genetics 159: $1271-1281$.

Tanurdzic M and Banks J. 2004. Sex-determining mechanisms in land plants. Plant cell 16: 561-571.

Verma SC and Selivan PM. 2001. Intraspecific variation in sporesize of homosporous ferns and its implications on fern mating systems. Bionature 21: 1-9.

Manuscript received on 19 November 2012 and revised on 10 December 2012 